\title{
Estado del arte y elementos del reconocimiento automático de imágenes del cerebro
}

\author{
Keila Vasthi Cortés-Martínez, Manuel Mejía-Lavalle
}

Centro Nacional de Investigación y Desarrollo Tecnológico, Departamento de Ciencias

Computacionales, Cuernavaca, Morelos, México

\{keila.cortes, mlavalle\}@ cenidet.edu.mx

\begin{abstract}
Resumen. El área de la Inteligencia Artificial ha incursionando en el ámbito médico con el propósito de apoyar en el reconocimiento automático de imágenes del cerebro. Una de las enfermedades que ha ido en aumento en la última década es la Enfermedad Cerebro Vascular, cuyo conjunto de enfermedades, afectan los vasos sanguíneos cerebrales. Dados los efectos que puede causar este tipo de enfermedad, es importante caracterizar las fuentes que generan imágenes médicas y los procedimientos empleados para mejorarlas e interpretarlas. El documento describe los avances reportados en la literatura en relación a las fuentes que obtienen las imágenes y las etapas seguidas para su reconocimiento. El trabajo considera que existe la oportunidad de mejorar o desarrollar más algoritmos avanzados, que ofrezca un nivel más alto en la detección de enfermedades en imágenes del cerebro.
\end{abstract}

Palabras clave: enfermedad cerebro vascular, reconocimiento de imágenes médicas, inteligencia artificial, técnicas de visión artificial.

\section{State of the Art and Elements of Automatic Image Recognition of the Brain}

\begin{abstract}
Artificial Intelligence area has penetrated the medical field with the purpose of supporting the automatic recognition of brain imaging. One of the diseases that has been increasing in the last decade are Brain Stroke, whose set of diseases, affect the cerebral blood vessels. Given that effects that can cause this type of disease, it is important to characterize the sources that generate medical images and the methods used to improve and interpret them. This paper describes the advances reported in the literature in relation to the sources that obtain the images and the stages followed for their recognition. Moreover considers that there is an opportunity to improve and development of advanced algorithms, which will offer a higher level in the detection of diseases in brain images.
\end{abstract}

Keywords: brain stroke, medical image recognition, artificial intelligence, artificial vision techniques. 


\section{Introducción}

En el año 2001, la Secretaría de Salud de México [1], ubicó a las Enfermedades Cerebro-Vasculares (ECV), en el sexto lugar dentro de las diez principales causas de muerte en México. En el año 2014, la Organización Panamericana de la Salud [2], ubicó a las ECV en el segundo lugar, del Índice de enfermedades que incapacita a los adultos, lo cual indica que en el lapso de una década, la ECV se convirtió en un serio problema de salud.

Las ECV ocurren cuando hay afectaciones en los vasos sanguíneos del cerebro, provocando la disminución repentina de flujo sanguíneo o el rompimiento de ellos, haciendo que la sangre se esparza entre los espacios que rodean a las células cerebrales, dando lugar a dos tipos principales de términos: isquémico y hemorrágico [3]; se caracterizan por presentarse sin aviso y causan secuelas desde leves hasta severas: como parálisis, problemas de raciocinio, derrames, coordinación motora e incluso la muerte [4].

Las ECV pueden diagnosticarse mediante el uso de imágenes médicas obtenidas por medio de tomografías computarizadas, ultrasonidos (como el Doppler), electroencefalogramas, imágenes de resonancia magnética, etc. [3] El diagnóstico por interpretación de imágenes es invaluable, ya que se trata de un método no invasivo para la detección de la enfermedad. El reconocimiento de imágenes médicas de forma automatizada reduce el tiempo en la evaluación y minimiza errores en el diagnóstico y tratamiento de enfermedades [5].

Actualmente la inteligencia artificial aborda el tratamiento y análisis de imágenes médicas, desarrollando técnicas o modelos de aprendizaje automático y de reconocimiento de patrones, tales como la lógica difusa, red neuronal artificial (RNA) [6], k-means, entre otros [7], teniendo un gran éxito en la medicina como apoyo en la toma de decisiones para un diagnóstico más certero, representando uno de los avances más importantes de los últimos años, puesto que hoy en día se pueden examinar distintas actividades del cerebro al mismo tiempo (su función metabólica, eléctrica o cognitiva) [7].

Por la importancia del tema se hace necesario establecer una sinergia entre la disciplina médica y la disciplina de las ciencias de la computación; por lo tanto en este trabajo se presentan los estudios enfocados en el uso de herramientas computacionales para el tratamiento y diagnóstico de imágenes médicas. La literatura consultada corresponde a 79 artículos (Tabla 1).

Tabla 1. Literatura de países citados en el uso de imágenes médicas del cerebro (elaboración propia).

\begin{tabular}{l|c|c|c|c|c|c}
\hline PAÍSES & TC & MRI & EEG & Doppler & $\begin{array}{c}\text { En } \\
\text { general }\end{array}$ & TOTAL \\
\hline Alemania & & {$[60]$} & & & & 1 \\
\hline Argentina & & & & {$[14]$} & & 1 \\
\hline Bélgica & & {$[52]$} & & & & 1 \\
\hline Canadá & & & & & {$[47][67]$} & 2 \\
\hline
\end{tabular}


Estado del arte y elementos del reconocimiento automático de imágenes del cerebro

\begin{tabular}{|c|c|c|c|c|c|c|}
\hline PAÍSES & TC & MRI & EEG & Doppler & $\begin{array}{c}\text { En } \\
\text { general }\end{array}$ & TOTAL \\
\hline China & & [70] & [31] [36] & & & 3 \\
\hline Colombia & & & [28] & & & 1 \\
\hline Cuba & & & & [16] & & 1 \\
\hline España & & [10] & $\begin{array}{c}{[4][3][9]} \\
{[59][77]}\end{array}$ & [18] & [85] & 8 \\
\hline $\begin{array}{l}\text { Estados } \\
\text { Unidos }\end{array}$ & [34] [81] & $\begin{array}{c}{[11][34][43]} \\
{[45]}\end{array}$ & $\begin{array}{l}{[20][22]} \\
{[26][29]} \\
{[32][71]} \\
\end{array}$ & & [23] [83] & 14 \\
\hline India & $\begin{array}{l}{[12][13]} \\
{[30][40]}\end{array}$ & $\begin{array}{c}{[25][33][38]} \\
{[39][62][40]} \\
{[48][49]}\end{array}$ & [21] & & $\begin{array}{l}{[27][58]} \\
{[74][76]}\end{array}$ & 17 \\
\hline Irán & & & & & $\begin{array}{c}{[53]} \\
{[64][65]} \\
\end{array}$ & 3 \\
\hline Iraq & [35] & & & & [74] & 2 \\
\hline Israel & [66] & & & & & 1 \\
\hline Corea & [44] & & & & & 1 \\
\hline Malasia & $\begin{array}{c}\text { [41] [56] } \\
{[80]}\end{array}$ & & [24] & & [78] & 5 \\
\hline México & [55] & & $\begin{array}{c}{[8][72]} \\
{[82]} \\
\end{array}$ & [17] & & 5 \\
\hline $\begin{array}{l}\text { Nueva } \\
\text { Zelanda }\end{array}$ & & & [69] & & & 1 \\
\hline Polonia & [57] [75] & & & & & 2 \\
\hline $\begin{array}{l}\text { Reino } \\
\text { Unido }\end{array}$ & & & & & [61] & 1 \\
\hline Rumania & & & & & [6] [79] & 2 \\
\hline Sri Lanka & [37] & & & & & 1 \\
\hline Sudán & [46] & & & & & 1 \\
\hline Suiza & & [5] & & & & 1 \\
\hline Taiwán & [36] & & & & & 1 \\
\hline Túnez & & & & & 3 & 1 \\
\hline Turquía & & [42] & [73] & & & 2 \\
\hline TOTAL & 18 & 18 & 21 & 4 & 18 & 79 \\
\hline
\end{tabular}

\section{Fuentes de imágenes médicas cerebrales}

Para diagnosticar las ECV es necesario el uso de imágenes médicas. Una imagen médica es aquélla que resulta del conjunto de técnicas y procesos usados para crear imágenes del cuerpo humano, o partes de él, con propósitos clínicos, es decir imágenes que buscan revelar, diagnosticar o examinar enfermedades, o bien, servir sólo para propósitos científicos médicos [9]. El uso de tecnologías generadoras de imágenes 
cerebrales es uno de los avances más significativos en la medicina [9]. A continuación se describen las ventajas y desventajas de cada una de ellas.

\subsection{Ultrasonido Doppler}

Es una herramienta ultrasonográfica rápida, fácil de usar, de bajo costo, indolora y no utiliza radiación ionizante; ayuda a visualizar el funcionamiento de los vasos cerebrales (dirección y velocidad del flujo sanguíneo); detecta si existe sangrado intracraneal u obstrucción así como tejidos blandos $[14,15,16]$. El ultrasonido Doppler ha sido utilizado durante procedimientos quirúrgicos como un método auxiliar en la valoración del flujo vascular en tiempo real [17] (Fig. 1).

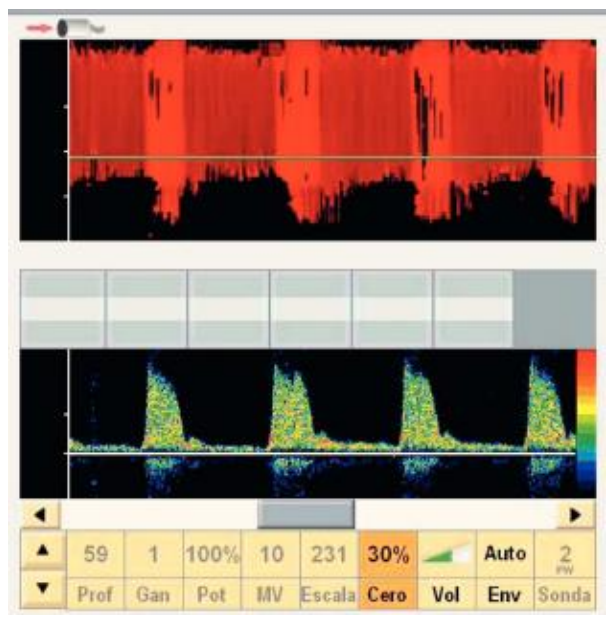

Fig. 1. Arteria cerebral media con alta presión intracraneal captada por ultrasonido Doppler [18].

\subsection{Imágenes de resonancia magnética (MRI)}

Las imágenes de resonancia magnética (MRI) contribuyen a la detección, diagnóstico y tratamiento de diversas patologías y enfermedades; una de sus ventajas es que tienen gran capacidad de distinguir entre materia gris y blanca del cerebro (Fig. 2). La MRI se realiza dentro de un campo magnético (sin radiación), y junto con la radio frecuencia altera el alineamiento natural de los átomos de hidrógeno en el cuerpo [10], por lo que resulta no invasiva para el paciente. Durante la obtención de las MRI, existe ruido y variación paramétrica que presentan problemas especiales en los procesos de automatización, por lo que es importante evaluar cada imagen y calcular cada uno de sus pixeles con el fin de suavizar los datos [11].

\subsection{Tomografía computarizada}

La tomografía computarizada (TC) crea una serie de imágenes transversales del cerebro, que precisan la detección de anomalías pequeñas; permite encontrar tumores 
y otras enfermedades con mayor facilidad, reduciendo tiempo y costo en el diagnóstico [12]. Se realiza mediante la aplicación de rayos-X, obteniendo múltiples imágenes detalladas en distintos ángulos, lo cual permite diversas vistas del cerebro (Fig. 2).

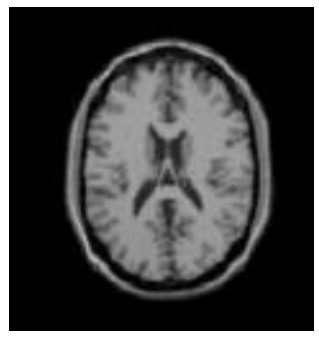

(a)

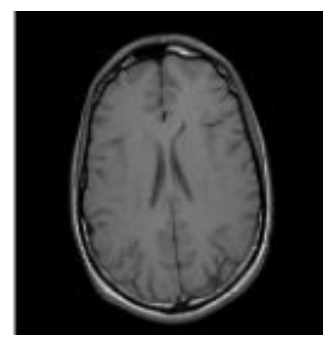

(b)

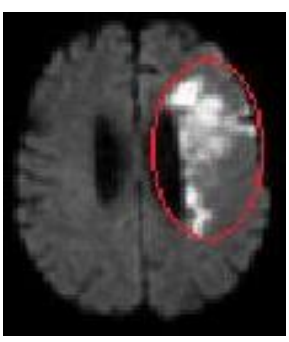

(c)

Fig. 2. Imágenes cerebrales: (a) MRI normal, (b) TC normal, (c) TC anormal [13].

\subsection{Electroencefalogramas}

El electroencefalograma (EEG) representa las actividades eléctricas del cerebro llamadas impulsos durante un corto período de tiempo, las cuáles se registran a partir de electrodos colocados en el cuero cabelludo obteniendo una representación de líneas en múltiples canales a lo largo del tiempo [19, 20]. Esta técnica de diagnóstico requiere de profesionales capacitados para su interpretación lo que resulta tediosa y lenta, no obstante es una importante herramienta no invasiva que diagnostica, evalúa, monitorea y predice una ECV [21, 22] (Fig. 3); cuando percibe una ECV del tipo isquémico, el EEG es muy sensible ya que se observan diversos cambios anormales en los patrones debido al bajo nivel de oxígeno que presenta el cerebro [23].

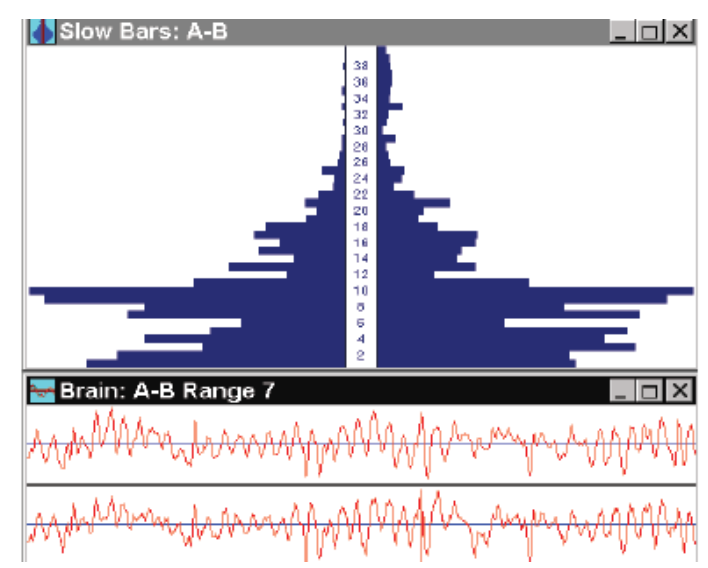

Fig. 3. Señal de ondas cerebrales con EGG [24].

Las imágenes obtenidas mediante herramientas computacionales pasan por varias etapas para llegar a la interpretación y la obtención de un diagnóstico de una enfermedad cerebro vascular (Fig. 4). 


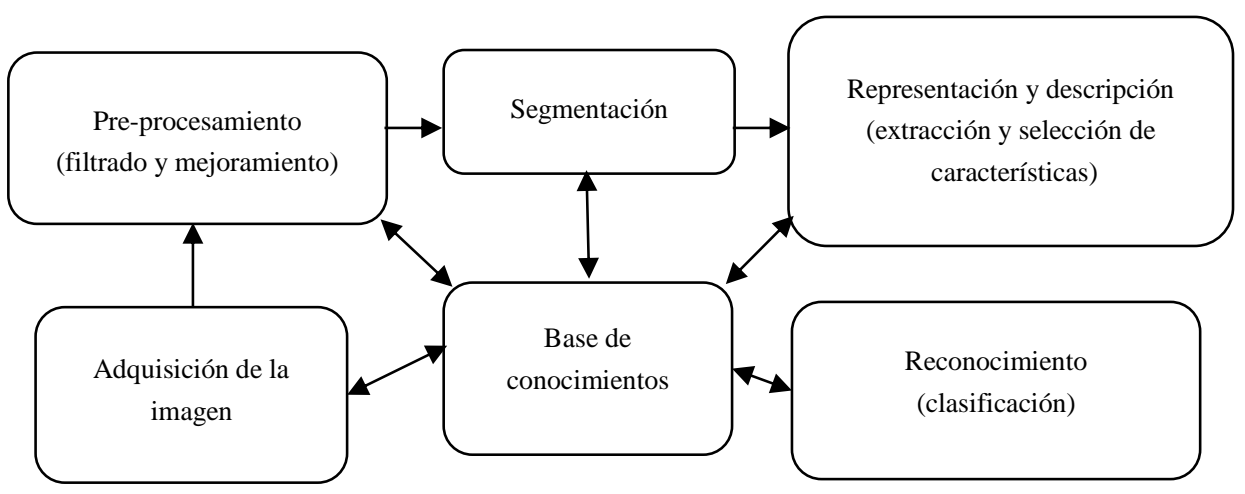

Fig. 4. Etapas comunes (no aplicables en algunos casos) en la interpretación de imágenes médicas [8].

\section{Etapas en la interpretación de imágenes médicas}

En esta sección se describen brevemente algunas técnicas o métodos para el preprocesamiento, segmentación, descripción y reconocimiento de características de imágenes médicas cerebrales, presentados en artículos científicos orientados a la neurociencia con base en la inteligencia artificial.

\subsection{Pre-procesamiento de los datos}

En la literatura se identificaron 31 referencias; en estas se indica que el preprocesamiento de una imagen consiste en aplicar técnicas para la búsqueda de datos a partir de una imagen original, mejorando sus características en otra imagen, con la finalidad de ser analizadas y que permitan realizar operaciones a partir de información hallada; aplicando esto a imágenes médicas, su papel es crucial para el tratamiento y diagnóstico de enfermedades. Las imágenes originales contienen "detalles ajenos" (o artefactos) cuando presentan algún tipo de ruido o moteado, por lo que el resultado del diagnóstico del médico al paciente puede errar. Uno de los objetivos del preprocesamiento es el de normalizar o estandarizar los datos de una imagen previa. En algunos casos [11] no es necesaria esta etapa; en otros, por ejemplo [25], los artefactos presentes en las imágenes médicas suelen eliminarse manualmente, algunos de estos son derivados de los aparatos con los que se toman las muestras [21,26]. Muchos autores coinciden en que una de las técnicas más importantes en esta etapa es el filtrado de imágenes [27], que ayuda en el mejoramiento de una imagen con el fin de adquirir información; dentro de la literatura citada se dividen en 2 grupos:

1) De acuerdo a su dominio de frecuencia: Este tipo de filtrado es usado comúnmente en los EEG, por ejemplo usando la transformada de Fourier [21,24,28,29], se elimina la presencia de ruido ocasionados por parpadeos oculares, actividad muscular, etc. [30]. Para artefactos ocasionados por los aparatos empleados, se usa el filtro paso de banda [31], para estandarizar datos se utiliza el filtrado pasa-bajo [32]. 
2) De acuerdo a su dominio espacial: Se identifican frecuentemente en el preprocesamiento de MRI y TC, los filtros destacados son: medio [27], Laplaciano [27], máximo [33], Gaussiano [13,27,36] y el filtrado mediano, los cuáles ayudan en la eliminación de ruido sin afectar la nitidez de la imagen médica [25,27,33,34,35],

En diversos artículos, se mencionan otras técnicas recurrentes usadas en esta etapa como: los métodos de binarización [13,35,37,38,39,40], eliminación de ruido [40], ecualización(o mejoramiento del contraste) $[27,38,39,40,46]$, curva cúbica aplicada al contraste [36], corrección de ángulo inclinado [13], detección simétrica del eje [41], redimensión(como normalización en el tamaño de las imágenes) [13,37,42,43,44], intensidad de la imagen [40,45] y transformada wavelet [27]. Alhawaimil y otros investigadores $[35,45,48]$ describen un método de pre-procesamiento de cuatro pasos para tratar la naturaleza patológica del tejido cerebral en imágenes de TC:

1) Se analiza la imagen RGB (modelo de color) de una tomografía computarizada y se convierte a una imagen en escalas de grises $[10,42,45]$;

2) Se aísla la sección del tejido cerebral escaneado, mediante el cálculo del área de la materia blanca del cráneo [35,40], por medio de algún software [43] o mediante la técnica de crecimiento de regiones [35,47]. Aquí también se puede hacer una detección de bordes usando Canny [48].

3) Se enmascara la imagen mediante un generador lógico OR para eliminar ruido con un filtro mediano de ventana [35].

4) Por último se utilizan operaciones morfológicas de erosión, dilatación y expansión para "limpiar" la imagen eliminando imperfecciones de la parte que corresponde al cráneo [13,35,36,39,42]; o mediante la técnica de Otsu [46].

En el caso de los electroencefalogramas, al pre-procesamiento de las señales se agregan dos métodos adicionales: la técnica Welch [24] y la ventana de Haming. Dentro de esta etapa las redes neuronales han tenido buenos resultados durante esta etapa, las más comunes son: las redes de función de base radial (RBF) [28], de retropropagación (FeedFoward) [42,49] y las multi-layer perceptron (MLP) $[28,32,50]$.

\subsection{Segmentación de los datos}

En la literatura consultada se identificaron 26 referencias. Después de "limpiar" o pre-procesar las imágenes se prosigue a hacer una segmentación, que es el proceso mediante el cual se divide la imagen en regiones con propiedades similares, tales como color, textura, brillo, contraste, niveles de grises; dentro de las imágenes médicas, la segmentación se define como la partición de una imagen en regiones constituyentes sin traslape, las cuáles son homogéneas con respecto a alguna característica como una intensidad o una textura $[51,52,53]$. Su objetivo radica en el estudio estructural e identificación de regiones de interés (tumores, lesiones y otras anomalías), siendo esto una tarea difícil, ya que este tipo de imágenes son de naturaleza compleja, ocasionando que seleccionar un algoritmo de segmentación dependa de diferentes aspectos a considerar ya que cada sistema de imágenes médicas tiene sus propias limitaciones; sin embargo, diversos métodos de segmentación se pueden aplicar a una amplia gama de 
datos [12,52,54]. Por ejemplo, Diyana et al. [56] segmenta el área intracraneal con técnicas ROI en las imágenes de tomografía computarizada usando fuzzy C-Means y el método de umbral de Otsu para definir importantes estructuras cerebrales y otras regiones que pueden contener anomalías; para Alhawaimil [34], los mejores métodos de segmentación son: dilatación y erosión; Sangeetha y Shahin [58] alimenta una RNA con el algoritmo K-NN; Yeu-Sheng et al. [36] aplica un algoritmo de crecimiento de regiones con filtro Gaussiano y la técnica Canny para obtener un mapa de bordes de las áreas importantes dentro de una tomografía computarizada original, separando la materia gris y resaltando el área de una ECV. A manera introductoria, investigadores $[12,40,54]$, describen que los métodos de segmentación se dividen en cinco grandes grupos (Tabla 2).

En una revisión de Ramírez y Chacón [8] se divide a las técnicas de segmentación de la siguiente manera, aunque se enfocan especialmente en las RNAs:

Tabla 2. Métodos de segmentación.

\begin{tabular}{c|l}
\hline Basados en: & \multicolumn{1}{c}{ Descripción } \\
\hline Umbrales & -Umbralización (tresholding) [12,34,35,39,40,55] \\
& - Textura y por niveles de grises (subdivisión propuesta por Aggarwal et \\
& al.[54]) \\
& - Manuales, semi-automatizadas o automatizadas(sub-categoría propuesta \\
& en [40]) \\
& - Método de umbralización óptimo para región de interés (ROI) [56,57] \\
& -Otsu [56] \\
\hline Regiones & -Crecimiento de regiones: [12,36,40,55] \\
{$[3,14,48]$} & -Crecimiento de cuencas (Watershed): [37,40] \\
\hline Bordes[48] & -Canny: [12,36,55] \\
& -RNA Convolucional (CNN), Red Neuronal Pulso-Acoplada (PCNN), \\
& Mapa Auto-organizado de Kohonen (SOM), Redes Neuronales \\
& Oscilatorias (ONN) [8] \\
\hline Pixeles & -Fuzzy C-Means: [6,11,40] \\
& -Campo Aleatorio de Markov: [40] \\
& -RNA: [12,34,40] \\
& -RNA Multi-Layer Perceptron: [33] \\
\hline Agrupación & -Modelos Paramétricos Deformables: [40] \\
& -Modelos Geométricos Deformables: [40] \\
& -Clustering: [12] \\
\hline
\end{tabular}

- De bordes y formas: Se crea una imagen binarizada, extrayendo las regiones más representativas a partir de contornos de objetos en la imagen, la RNA más versátil fue la red neuronal convolucional (CNN) usada en imágenes con formas irregulares [8].

- Segmentación de Color y textura: Se hace una partición de la imagen en regiones que agrupan colores similares e intensidades de grises. Las variantes de las RNAs con mejores resultados son: mapa auto-organizado de características (SOFM), variante de la SOM paramétrica (PSOM), variación de SOM con un algoritmo genético (SOFT-NN), SOM de estructura adaptiva (SASOM), así como algoritmos de mezclas gaussianas (GMM) y oscilaciones sincronizadas con CNN (SON) [53]. 
- Movimiento: A partir de una secuencia de imágenes se detectan objetos, según la literatura analizada los más eficientes son: self organization background substraction (SOBS), fuzzy clustering neural network (FCNN), CNN de tiempo discreto (DTCNN) y time delay neural network (TDNN). Anusha et al. y Ceballos $[21,28]$ utilizan el método de "ventana deslizante" (sliding window segmentation, en inglés) dentro de encefalogramas, para dividir las señales producidas en épocas y segmentarlas en canales [31,59].

Otras técnicas de esta etapa son: operaciones morfológicas (de erosión y dilatación) [34,37,38], binarización [60], filtro mediano [35,36], euclidiana [62], inferencia bayesiana [61], así como las RNAs [53] descritas como técnicas rápidas y precisas en la segmentación de una imagen cerebral [11]; Cárdenas [63] utiliza redes artificiales pulso-acopladas (PCNN, por sus siglas en inglés) con metodologías clásicas, como entropía cruzada y la métrica de correspondencia de pixeles (PCM, por sus siglas en inglés) para detección de bordes. Shanbehzadeh y Rastgarpour [64,65] dividen las técnicas de segmentación de acuerdo a su aplicación en cuatro grupos:

1) Métodos Híbridos. Son técnicas de Inteligencia Artificial combinadas con algoritmos de aprendizaje automático y operadores de procesamiento de imágenes, lo que hace que sean semi-automáticos.

2) Sistemas expertos. Aquí el conocimiento de los expertos se utiliza en forma de reglas, modelos o atlas [56].

3) Métodos de procesamiento de imágenes. Se utilizan características múltiples de diferentes imágenes para aumentar la precisión en cada una de las categorías a analizar de una imagen [66].

4) Métodos Multiespectrales o multimodales. Se utilizan múltiples imágenes de un órgano para tener características de variantes en la imagen, y realizar una fusión para la obtención de plantillas [67.]

La comparación de la literatura indica que la segmentación basada en RNAs y por umbralización son los métodos más frecuentes debido al aislamiento de pixeles que realizan; partiendo de esto, Gordillo et al. [68] realiza un resumen de las ventajas y desventajas que poseen estos métodos en la aplicación de imágenes de resonancia magnética con presencia de tumores cerebrales.

\subsection{Representación y descripción (extracción y selección de características)}

De un total de 79 artículos, 26 referencias contienen esta etapa y se mencionan 45 técnicas. Para representar y describir de manera adecuada una imagen es necesario representar la naturaleza de la misma en forma numérica, para ello se extraen y se seleccionan atributos o rasgos más relevantes y con menor redundancia, que ayuden a facilitar la visualización y comprensión de los datos. Por ejemplo, el diseño utilizado para la alimentación de árboles de decisión en [46] se formó de un conjunto de 14 atributos obtenidos de las características sugeridas por especialistas, formando un conjunto de referencia que ayudan en la descripción de ECV isquémica; algo parecido se plantea en [27], donde usando el algoritmo K-NN y clasificadores bayesianos, para extraer características de una imagen médica se toman decisiones con respecto a la 
patología de una estructura o tejido ateromatosa. Para el diagnóstico de la enfermedad de Alzheimer [47], se hace un etiquetado de voxels a partir la segmentación hipo campal dimensional o mediante una red neuronal pulsante ( $\mathrm{SNN}$, por sus siglas en inglés) [69]. Para representar la actividad normal y anormal del cerebro, se usan diversas técnicas como: características binarias, análisis de componentes principales, análisis discriminante lineal, código de cadena, transformación de características invariante a escala, filtrado Gabor, distancia euclidiana, red neuronal artificial, máquinas de soporte vectorial [13], matriz de co-ocurrencia de niveles de grises [39,40], descriptores de textura Haralick [44], umbralización (cambio en los niveles de intensidad); Stier et al. [43] utiliza una red neuronal convolucional (CNN) para generar un modelo predictivo. Tyan et al. [36], Balasooriya y Perera [37] usan los métodos de Otsu, filtro anisotrópico, máscara gaussiana, métodos morfológicos (erosión y dilatación) para determinar el área cerebral izquierda o derecha, en la que sucedió la ECV y eliminar ruido de fondo; Haiyan Zhang [70] usa un modelo de contorno geométrico activo mejorado. En imágenes de electroencefalogramas, se captura la duración y el tamaño de la amplitud de las ondas mediante la técnica de ventana deslizante [28,32], la descomposición wavelet[44], el dominio de frecuencias características, transformación de wavelet discreta, la longitud de la transformada de Fourier rápida [21,24,59,71,72,73], y la transformada de ondula discreta de superposición máxima (MODWT) [72]; Ceballos [28] con una RNA multi-layer perceptron crea una matriz de características y patrones del electroencefalograma. Otras técnicas mencionadas en general, para realzar el contraste de la imagen en búsqueda de tumores son: técnicas de media, valores de histograma [44], operaciones morfológicas (dilatación y erosión) [11,25,38,42,62]; curtosis [28,33], matriz de co-ocurrencia de niveles de gris[74,75], desviación estándar, asimetría [57], entropía de Shanon [32], algoritmo Gabor [44], el algoritmo k-means [33]; máscara binaria basada en regiones(max-min)[38], así como diversas técnicas de minería de datos [44]. Vishal et al. [76] menciona a la red neuronal convolucional (CNN) como la mejor técnica para extraer características, comparada con máquinas de soporte vectorial y árboles aleatorios; Boublil et al. [66] extrae características de diferentes versiones de una misma imagen de tomografía computarizada mediante una RNA, para después fusionarlas y mejorar su resolución; algunos métodos de fusión se mencionan en [67]. En esta sección generalmente se presentan algunos problemas al escoger características importantes de la imagen, debido a que estas deben de presentar el menor número de invariancia respecto a la imagen original.

\subsection{Interpretación y reconocimiento}

De un total de 79 artículos, 15 tienen referencia de esta etapa, mencionándose 11 técnicas y 5 algoritmos. La interpretación y reconocimiento de las características de una imagen médica sirven para dar un significado a los datos obtenidos de la imagen. En esta etapa se utilizan algunos algoritmos de agrupamiento y métodos de clasificación, los cuáles unen espacios característicos de un conjunto de datos, mediante intensidades de la imagen. En ocasiones, esta etapa se realiza de manera "manual", con la ayuda de especialistas [3,77], que clasifican y diagnostican una ECV mediante un algoritmo de diagnóstico etológico del ictus cerebral, o [35] a partir de una comprobación visual de 
la región con lesiones. Lashari y Ibrahim[78] relatan que la clasificación de imágenes médicas se centra principalmente en el uso de algoritmos eficientes de minería de datos y técnicas de visualización, en este caso, se propone un algoritmo de clasificación basado en las nociones de teoría de conjuntos blandos, y se utilizan técnicas como la wavelet y Gabor, entre otras. Para tener una mejor interpretación de una imagen médica, se suelen afinar detalles obtenidos en etapas anteriores, por ejemplo Lee et al. [44] realizan un método para suavizar imágenes de tomografía computarizada mediante características obtenidas de una distancia ponderada gaussiana, reconstruyendo el cerebro humano en 3D y encontrando la ECV. Hussein Ali et al. [35], obtuvieron buenos resultados en el reconocimiento de cerebros enfermos, haciendo que los valores de gris de la parte anormal brillaran más. Karthikeyan y Dr.Ezhilarasi [25] interpretan lesiones mediante el crecimiento de regiones. RosemehahWan et al. [24] predice la actividad normal y anormal del cerebro en un encefalograma con K-NN (k-nearest neighbors); por medio de un análisis usando RNAs y máquinas de soporte vectorial, Menaka y Kanchana [13] desarrollan una base de datos con lesiones cerebrales; Yahiya et al. [46] emplea árboles de decisión y algoritmos de aprendizaje (k-means) para presentar un modelo de clasificación de ECV isquémica. En $[61,78,79]$ se realizan algunas métricas de similitud con el fin de reconocer y clasificar anomalías dentro de las tomografías computarizadas, así como detección de anomalías basadas en reglas, en [80] se hace una colorización en base a un mapa de colores estándar. Chawla et al. [81] usa una metodología de tres pasos: primero mejora la imagen, después hace una detección de la simetría cerebral y al último hay una clasificación de cortes anormales, similar a la realizada por Bhaiya y Verma [49] donde se utilizan técnicas como la transformación wavelet, el análisis de componentes principales y métodos de aprendizaje supervisado como el algoritmo de retropropagación, red neuronal de funciones radiales y un vector de aprendizaje de cuantización. Toprak [73], Menaka y Kanchana [13] usan al final de la clasificación RNAs con algoritmos basados en en Levenberg-Marquardt, BFGS Quasi-Newton (Broyden-Fletcher-Goldfarb-Shanno with Quasi-Newton, en inglés) y máquinas de soporte vectorial. Otras técnicas descritas en esta etapa son: la técnica de características binarizadas, el algoritmo de código de cadena y la técnica de transformada de características invariantes, usando una distancia euclidiana [13]. Así mismo, las RNAs [55] se mencionan en distintos artículos de la literatura consultada, principalmente:

- Backpropagation: Útil en reconocimiento de tumores cerebrales o anomalías pequeñas [42], considerada como una gran clasificadora [28].

- FeedFoward: [59,72].

- $\quad$ RNA sin especificar: [37,55] red neuronal con método de cuenca [21,26] como modelo de predicción en la detección de una ECV recurrente.

- Multi-Layer Perceptron (MLP): útil al clasificar los tejidos a dos clases de normales y anormales [39].

En la revisión realizada por Ramírez y Chacón [8] las técnicas de clasificación usando RNA más relevantes son: teoría de resonancia adaptiva (ART), multi-layer perceptron, red neuronal con funciones de base radial y red neuronal probabilística. De acuerdo a Ramírez-Quintana et al. [82], la RNA de teoría de resonancia adaptativa tuvo 
muchos resultados favorables en el reconocimiento al igual que la red neuronal convolucional(CNN)[83], red neuronal probabilística y mapa auto-organizado de Kohonen (SOM, por sus siglas en inglés), debido a su flexibilidad en el procesamiento de color, análisis de movimiento y detección de formas.; la red neuronal con funciones de base radial fue la más exitosa en la segmentación de MRI; así mismo preveen que el uso de éstas RNAs sean una tendencia, especialmente en las SOM, CNN y Pulsantes; por lo que experimentar con redes neuronales pulsantes, augura buenos resultados (en especial en la clasificación). Actualmente existen algunas herramientas de software de código abierto utilizadas en la interpretación de imágenes médicas en general, en [84] se realiza una comparación de distintas aplicaciones, de las cuáles sólo SPM, FSL y DTI-TK ayudan en imágenes relacionadas con las ECV, con una tendencia por las que analizan Imágenes de Resonancia Magnética, como se describe en la Tabla 3.

Tabla 3. Fragmento de [84], sobre herramientas de software utilizadas en la interpretación de imágenes médicas.

\begin{tabular}{|c|c|c|c|c|c|c|}
\hline \multicolumn{2}{|c|}{$\begin{array}{l}\text { Herramientas de } \\
\text { Procesamiento de } \\
\text { Imágenes }\end{array}$} & $\mathrm{SPM}^{1}$ & $\mathrm{FSL}^{2}$ & ELASTIX $^{3}$ & MITK $^{4}$ & $\begin{array}{l}\text { DTI- } \\
\text { TK }^{5}\end{array}$ \\
\hline \multicolumn{2}{|c|}{ Última Versión } & 12 & 5.0 & 4.7 & 3 & 3 \\
\hline \multicolumn{2}{|c|}{ Publicados } & 2014 & 2014 & 2014 & 2014 & 2011 \\
\hline \multirow{5}{*}{$\begin{array}{c}\text { Imágenes } \\
\text { Soportada } \\
\mathrm{s}\end{array}$} & MRI & $\mathrm{X}$ & $\mathrm{X}$ & $\mathrm{X}$ & $\mathrm{X}$ & $\mathrm{X}$ \\
\hline & $\mathrm{TC}$ & $\mathrm{X}$ & & $\mathrm{X}$ & $\mathrm{X}$ & \\
\hline & EEG & $X$ & & $\mathrm{X}$ & $\mathrm{X}$ & $\mathrm{X}$ \\
\hline & Ultrasonido & & & $\mathrm{X}$ & $\mathrm{X}$ & $X$ \\
\hline & $\begin{array}{l}\text { imágenes de } \\
\text { 3D }\end{array}$ & & & $X$ & $\mathrm{X}$ & $\mathrm{X}$ \\
\hline \multirow{3}{*}{ Funciones } & Segmentación & $X$ & & $\mathrm{X}$ & $X$ & \\
\hline & Visualización & & & $\mathrm{X}$ & $X$ & \\
\hline & $\begin{array}{l}\text { Reconstrucci } \\
\text { ón }\end{array}$ & $\mathrm{X}$ & $\mathrm{X}$ & $\mathrm{X}$ & & $X$ \\
\hline \multirow{5}{*}{$\begin{array}{c}\text { Lenguajes } \\
\text { del } \\
\text { Sistema }\end{array}$} & $\mathrm{C}$ & $\bar{X}$ & & & $\mathrm{X}$ & \\
\hline & $\mathrm{C}++$ & & & $\mathrm{X}$ & & \\
\hline & $\mathrm{C \#}$ & & $\mathrm{X}$ & & & $\mathrm{X}$ \\
\hline & Java & & & & & $\mathrm{X}$ \\
\hline & $\begin{array}{l}\text { Código } \\
\text { Abierto }\end{array}$ & $\mathrm{X}$ & $\mathrm{X}$ & $\mathrm{X}$ & $\mathrm{X}$ & $\mathrm{X}$ \\
\hline \multirow{3}{*}{$\begin{array}{l}\text { Plataforma } \\
\text { s }\end{array}$} & Windows & $\mathrm{X}$ & $\mathrm{X}$ & $\mathrm{X}$ & $X$ & $\mathrm{X}$ \\
\hline & Linux & $X$ & $\mathrm{X}$ & $\mathrm{X}$ & $\mathrm{X}$ & $\mathrm{X}$ \\
\hline & Mac OS X & & & $\mathrm{X}$ & $\mathrm{X}$ & $\mathrm{X}$ \\
\hline
\end{tabular}

${ }^{1}$ Statistical Parametric Mapping; ${ }^{2}$ FMRIB Software Library; ${ }^{3}$ Elastix es un software de código abierto, basado en el conocido Insight Segmentation and Registration Toolkit (ITK); ${ }^{4}$ MITK Medical Imaging Interaction Toolkit; ${ }^{5}$ DTI-TK Conjunto de herramientas de normalización espacial que utiliza datos de neuro-imágenes.

\section{Discusión}

En la literatura revisada se identificó que las imágenes de resonancia magnética son herramientas eficientes para detectar de manera temprana una ECV isquémica, al 
basarse en campos magnéticos logran distinguir mejor la materia blanca de la gris y puede predecir una mayor variedad de ECV.

La tomografía computarizada es una técnica relativamente rápida en la creación de la imagen cerebral y tiene una buena precisión en la detección de ECV hemorrágicas; sin embargo, presenta una exposición a la radiación dado que utiliza rayos-X. El electroencefalograma es una fuente lenta en la obtención de imágenes cerebrales, sin embargo es utilizada principalmente para rectificar la presencia de alguna ECV, también se utiliza para detectar Alzheimer y la epilepsia.

El ultrasonido Doppler es una técnica que evalúa la dirección como la velocidad del flujo sanguíneo generando datos espectrales, en este estudio se encontró poca literatura, sin embargo debido a su naturaleza algunos autores han especulado que se pueden estudiar mediante redes neuronales. En la literatura consultada se encontró una mayor cantidad de estudios cuyo origen es el país de la India, seguido de Estados Unidos y España (Tabla 1).

También se muestra que el electroencefalograma se utiliza con mayor frecuencia, este dato debe ser interpretado con cautela, puesto que según lo descrito depende de las ECV que se desean buscar; en el estudio actual, las tomografías computarizadas y las imágenes de resonancia magnética obtuvieron la misma relevancia en cuanto a su uso, sin embargo las imágenes de resonancia magnética son menos invasivas. Las técnicas más utilizadas por etapa fueron:

- Pre-procesamiento: el Filtrado, las Operaciones morfológicas y la Binarización.

- Segmentación: las técnicas basadas en pixeles (Umbralización) y RNAs.

- Representación y Descripción: hubo variedad en la extracción de características dependiendo del tipo de imagen a analizar, así como de la selección de parámetros a estudiar, sobresaliendo las RNAs y filtros.

- Interpretación: las RNAs fueron las más utilizadas y con mejores resultados en comparación a otras técnicas.

\section{Conclusiones}

La literatura indica que con cada herramienta computacional y con cada enfermedad se definen métricas o parámetros, descriptores, técnicas, algoritmos y herramientas de software diferenciados para el tratamiento de imágenes. En la literatura consultada se describen 120 técnicas, 5 algoritmos y 5 herramientas de software.

Las técnicas de Umbralización, Filtrado y Redes Neuronales Artificiales aparecieron en todas las etapas. En los 79 estudios se identificaron 17 tipos de RNAs pero pocos estudios sobre redes pulsantes. Consideramos que este este tipo de red puede tener una buena efectividad en la extracción y selección de características de imágenes del cerebro.

Agradecimientos. Se agradece al Tecnológico Nacional de México por el financiamiento al proyecto 5683.16-P. 


\section{Referencias}

1. Secretaría de Salud: Programa de Acción: Enfermedades Cardiovasculares e Hipertensión Arterial. Secretaría de Prevención y protección de la Salud, México, D.F. (2001)

2. MÉXICO: Perfil de Enfermedades Cardiovasculares. Organización Panamericana de la Salud. Revista de la Facultad de Medicina de la UNAM, pp. 11 (2014)

3. Blanco-González, M., Arias-Rivas, S., Castillo-Sánchez. J.: Diagnóstico del accidente cerebrovascular isquémico. Unidad de Ictus, Área de Neurociencias, Servicio de Neurología, Hospital Clínico Universitario, Santiago de Compostela, España, pp. 49194923 (2011)

4. Monge-Pereira, E., Molina-Rueda, F.: Electroencefalografía como método de evaluación tras un ictus. Una revisión actualizada. Neurología (2014)

5. Rihiardi, J.: Machine Learning with Brain Graphs. IEEE Signal Processing Magazine, Vol. 30, pp. 58-70 (2013)

6. Rotariu, C., Costin, H.: Medical Image Processing by means of some Artificial Intelligence methods. Buletinul Institutului Politehnic Din Iasi (2013)

7. Fundación Española para la Ciencia y la Tecnología(FECYT): Viaje al Universo Neuronal. Unidad Didáctica (2007)

8. García, I.: Aportaciones a la Segmentación y Caracterización de Imágenes Médicas 3D. Capitulo II. Imágenes Médicas, Madrid (2010)

9. López, A.: Desarrollo de un sistema de análisis automático de perfusión en RMI de cerebro. Departamento de Tecnología Electrónica y de las Comunicacione, Madrid, España, (UAM) (2015)

10. Pukish, M. S., Wang, S., Wilamowski, B. M.: Segmentation of cerebral cortex MRI images with artificial neural network (ANN) training. Biomedical Engineering (2013)

11. Menze, B. H., Koen, V. L., Lashkari, D.: A Generative Probabilistic Model and Discriminative Extensions for Brain Lesion Segmentation With Application to Tumor and Stroke. IEEE Transactions On Medical Imaging, Vol. 35 (2016)

12. Fernández, J., Martínez-Sánchez, P., García-Rodríguez, R.: El eco-doppler color transcraneal en el estudio. Argentina, Neurología Argentina (2012)

13. DespotoviT, I., Goossens, B., Philips, W.: MRI Segmentation of the Human Brain: Challenges, Methods, and Applications. Hindawi Publishing Corporation Computational and Mathematical Methods in Medicine, pp. 23 (2015)

14. Lai, M.: Deep Learning for Medical Image Segmentation (2015)

15. Pappachen, A., Dasarathy, B.: A Review of Feature and Data Fusion with Medical Images, Canadá (2014)

16. Haiyan, Z., Jiafeng, L., Zixin, Z., Haiyun, L.: An automated and simple method for brain MR image extraction, BioMedical Engineering OnLine (2011)

17. Wei-Long, Z., Bao-Liang, Lu.: Investigating Critical Frequency Bands and Channels for EEG-Based Emotion Recognition with Deep Neural Networks2015, IEEE Transactions On Autonomous Mental Development, Vol. 7 (2015)

18. Yeu-Sheng, T., Ming-Chi, W., Chiun-Li, C., Yu-Liang, K., Ming-Sian, L., Hao-Yan, C.: Ischemic Stroke Detection System with a Computer-Aided Diagnostic Ability Using an Unsupervised Feature Perception Enhancement Method. Taiwan (2014)

19. Ceballos-Herrera, A.: Detección de epilepsia en señales de electroencefalografía, mediante el uso de RNA y MSV. Facultad De Ingeniería, Programa De Ingeniería Biomédica, Universidad Autónoma De Occidente, Santiago de Cali (2010) 
20. Scherle, C. E., Pérez-NellarI, J.: Utilidad del ultrasonido Doppler transcraneal en Neurología. Matamoros, La Habana, Cuba, Hospital Clinicoquirúrgico Hermanos Ameijeiras, Unidad de Ictus (2009)

21. Martínez-Vila, E., Fernández, M., Pagola, I., Irimia, P.:.Enfermedades Cerebrovasculares (2011)

22. Ling, G., Rivero, D., Seoane, J. A., Pazos, A.: Classification of EEG Signals Using Relative Wavelet Energy and Artificial Neural Networks. GEC, pp. 177-183 (2009)

23. Escudero, D.: Doppler transcraneal en el diagnóstico de la muerte encefálica. ¿Es útil retrasar el diagnóstico? Medicina Intensiva (2015)

24. Peek, N., Combi, C., Marin, R., Bellazzi, R.: Thirty years of artificial intelligence in medicine (AIME) conferences: A review of research themes (2015)

25. Alhawaimil, A.: Segmentation of Brain Stroke Image. International Journal of Advanced Research in Computer and Communication Engineering, Issue 9, Vol. 4, pp. 375-378 (2015)

26. Chawla, M., Sharma, S., Sivaswamy, J.: A method for automatic detection and classification of stroke from brain CT images. Engineering in Medicine and Biology Society, pp. 3581-3584 (2009)

27. Stier, N., Vincent, N., Liebeskind, D., Scalzo, F.: Deep Learning of Tissue Fate Features in Acute Ischemic Stroke. 2015 IEEE International Conference on Bioinformatics and Biomedicine (BTBM), California, pp. 1316-1321 (2015)

28. Younis, A., Ibrahim, M., Kabuka, M., John, N.: An Artificial Immune-Activated Neural Network Applied to Brain 3D MRI Segmentation. Journal of Digital Imaging (2008)

29. Carey, H. J.: EEG Interictal Spike Detection Using Artificial Neural Networks. III Department of Computer Science, Virginia Commonwealth University. Richmond, Virginia (2016)

30. Faught, E.: Current Role of Electroencephalography in Cerebral Ischemia. Dallas, TX, USA, Comments, Opinions, and Reviews, Vol. 24, pp. 609-613 (2016)

31. Zhenzhong, W., Lilin, G., Adjouadi, M.: Wavelet decomposition and phase encoding of temporal signals using spiking neurons. Neurocomputing, 173, pp. 1203-1210 (2016)

32. Abend, N. S., Dlugos, D. J., Clancy, R. R.: A review of long-term EEG monitoring in critically ill children with hypoxic-ischemic encephalopathy, congenital heart disease, ECMO, and stroke. 2, Pennsylvania , USA, J Clin Neurophysiol, Vol. 30, pp. 134-142 (2014)

33. Amarasinghe, K., Wijayasekara, D., Manic, M.: EEG Based Brain Activity Monitoring using Artificial Neural Networks. Idaho Falls, Idaho, USA, pp. 61-66 (2013)

34. Benayoun, M., Kohrman, M., Cowan, J., van Drongelen, W.: EEG, Temporal Correlations and Avalanches. Journal of Clinical Neurophysiology, Vol. 27, pp. 458-464 (2010)

35. Tajbakhsh, N., Jae-Shin, Y.: Convolutional Neural Networks for Medical Image Analysis: Full Training or Fine Tuning? (2016)

36. Claassen, J., Foreman, B.: Quantitative EEG for the detection of brain ischemia (2012)

37. Jyoti, G.: A Survey on Brain Stroke Detection in CT Images Using Image Segmentation. ISSUE 1, India, IJRECE, Vol. 4, pp. 41-43 (2016)

38. Menaka, R., Kanchana, R.: Computer Reinforced Analysis for Ischemic Stroke Recognition: A review. Indian Journal of Science and Technology, 8(35) (2015)

39. Gupta, N., Mittal, A.: Brain Ischemic Stroke Segmentation: A Survey (2014)

40. Mani-Maran, A., Saravanan, S.: Artificial Neural Networks (ANNs) for EEG Purging using Wavelet Analysis. International Journal of Electronics and Communication Engineering, Vol. 4, pp. 563-570 (2011) 
41. Karthikeyan, S., Ezhilarasi, M.: Automatic Stroke Lesion Segmentation From Diffusion Weighted Mri Images. Issue II, International Journal of Advanced Engineering Technology, Vol. VII, pp. 111-115 (2016)

42. Artificial Neural Network based Lesion Segmentation of Brain MRI. Tapas Si, Arunava De, Anup Kumar Bhattacharjee. 5, New York, USA : Foundation of Computer Science FCS, February de 2016, Communications on Applied Electronics, Vol. 4.

43. Sudharani, K., Sarma, T. C., Prasad, K.: Brain Stroke detection using K-Nearest neighbor and Minimum Mean Distance Technique. 2015 International Conference on Control,Instrumentation, Communication and Computational Technologies (ICCICCT) (2015)

44. Venkateswara-Reddy, B., Bhaskara-Reddy, P., Satish-Kumar, P., Siva-Reddy, S.: Developing an Approach to Brain MRI Image Preprocessing for Tumor Detection. International Journal of Research, Vol. 1, pp. 725-731 (2014)

45. Syed, A. Q., Narayanan, K.: Detection of Tumor in MRI Images Using Artificial Neural Networks. 9, September de 2014, International Journal of Advanced Research in Electrical, Electronics and Instrumentation Engineering, Vol. 3

46. Tijare, P. A., Vrushali, D. D.: CYST detection in MRI Brain Image. IMPACT: International Journal of Research in Engineering \& Technology, Vol. 2, pp. 129-138 (2014)

47. Bhaiya, L. P., Verma, V. K.: Classification of MRI Brain Images Using Neural Network. International Journal of Engineering Research and Applications, 5, Vol. 2, pp. 751-756 (2012)

48. Anusha, K S., Mathews, M. T., Puthankattil., S. D.: Classification of Normal and Epileptic EEG Signal using Time \& Frequency Domain Features through Artificial Neural Network. IEEE 2012 International Conference on Advances in Computing and Communications, pp. 98-101 (2012)

49. Sangeetha, C., Shahin, A.: Brain tumor segmentation using Artificial Neural Network. International Research Journal of Engineering and Technology, Vol. 2 (2015)

50. Nados, W. L., Kumar, R. D., Naser, M. A., Hussein, A. A.: Medical Images Classification by using Artificial Intelligence Techniques. Internal Journal of Scientific Engineering and Technology Research, Vol. 3, No. 34, pp. 6812-6816 (2014)

51. Priyanjana, S., Shagun, M., Surbhi, S., Jyotika, P.: Computer Aided Diagnosis Based on Medical Image Processing and Artificial Intelligence Methods. International Journal of Information and Computation Technology, Vol. 3, No. 9, pp. 887-892 (2013)

52. Vishal, T. V, Srinidhi, S., Srividhya, S., Sri-Vishnu, K., Swathika, R.: A Survey and Comparison of Artificial Intelligence Techniques for Image Classification and Their Applications. International Journal of Science and Research (IJSR), Vol. 5, No. 4 (2016)

53. Rastgarpour, M., Shanbehzadeh, J.: The Status Quo of Artificial Intelligence Methods in Automatic Medical Image Segmentation. International Journal of Computer Theory and Engineering, Vol. 5 (2013)

54. Soltanian-Zadeh, H., Jabarouti-Moghaddam, M.: Medical Image Segmentation Using Artificial Neural Networks. Artificial Neural Networks - Methodological Advances and Biomedical Applications, China, InTech, pp. 121-138 (2011)

55. Alyaa-Hussein, A., Shahad-Imad, A., Ihssan-Subhi, N.: Detection and Segmentation of Ischemic Stroke Using Textural Analysis on Brain CT Images. International Journal of Scientific \& Engineering Research, Vol. 6, No. 2 (2015)

56. Boublil, D., Elad, M., Shtok, J., Zibulevsky, M.: Spatially-Adaptive Reconstruction in Computed Tomography using Neural Networks. European Research Council under European Union's Seventh Framework Program (2014) 
57. Lee, H., Lee, J., Kim, N., Kim, S. J., Shin, Y. G.: Robust feature-based registration using a Gaussian-weighted distance map and brain feature points for brain PET/CT images. Computers in Biology and Medicine, Vol. 38, No. 9, pp. 945-961 (2008)

58. Diyana, W. M., Zaki, W., Faizal, M., Fauzi, A., Besar, R., Munirah, W. S. H., Ahmad, W.: Multi-level Segmentation Method for Serial Computed Tomography Brain Images. 2009 IEEE International Conference on Signal and Image Processing Applications, pp. 107-112 (2009)

59. Diyana, W. M., Zaki, W., Kong, C. R.: Identifying Abnormalities in Computed Tomography Brain Images Using Symmetrical Features. Selangor, Malasia, 2009 International Conference on Electrical Engineering and Informatics, Vol. 5, pp. 88-92 (2009)

60. Tan, T. L., Sim, K. S., Tan, C. K., Chong. A. K.: CT Image Enhancement by Colorization for Brain Infarct detection (2009)

61. Wan-RosemehahWan, O., Norfaiza, F., Mohd-Nasir, T., Rozita, J., Roshakimah-Mohd, I., Zunuwanas, M., Zaiton, S.: Brainwave Classification for Acute Ischemic Stroke Group Level Using k-NN Technique. 2014 Fifth International Conference on Intelligent Systems, Modelling and Simulation, pp. 117-120 (2014)

62. Lashari, S. A., Ibrahim, R.: A Framework for Medical Images Classification Using Soft Set. The 4th International Conference on Electrical Engineering and Informatics (ICEEI 2013), pp. 548-556 (2013)

63. Franco-Martínez, E. A.: Análisis digital de imágenes tomográficas sin contraste para la búsqueda de tumores cerebrales. Departamento de Computación, Centro de Investigación y de Estudios Avanzados del Instituto Politécnico Nacional, ciudad de México, Unidad Zacatenco, Diciembre (2011)

64. Juárez-Guerra, E., Alarcon-Aquino, V., Gómez-Gil, P.: Epilepsy Seizure Detection in EEG Signals Using Wavelet Transforms and Neural Networks. Conferences on computer, Information and Systems Sciences and Engineering (CCISSE), pp. 1-6 (2013)

65. Ramírez, J. A., Chacón, M. I.: Redes Neuronales Artificiales para el procesamiento de imágenes, una revisión de la última década. Revista De Ingeniería Eléctrica, Electrónica Y Computación, Vol. 9 (2011)

66. Ramírez-Quintana, J. A., Chacon-Murguia, M. I., Chacon-Hinojosa, J. F.: Artificial Neural Image Processing Applications: A Survey (2012)

67. Valderrama, J. A., Zárate, A., Martínez, B.: Utilidad Doppler En El Tratamiento De Aneurismas Cerebrales. México, Revista de especialidades médico quirúrgicas, Vol. 15, No. 4, pp. 189-195 (2010)

68. Kasabov, N., Capecci, E.: Spiking neural network methodology for modelling, classification and understanding of EEG spatio-temporal data measuring cognitive processes, Information Sciences, Vol. 294, pp. 535-575 (2015)

69. Ostrek, G., Nowakowski, A., Jasionowaka, M., Przelaskowski, A., Szopinski, K.: Stroke Tissue Pattern recognition based on CT texture analysis (2014)

70. Hachaj, T.: Artificial intelligence methods for understanding dynamic computer tomography perfusion maps (2010)

71. Jiang, J., Trundle, P., Ren, J.: Medical Image Analysis with Artificial Neural Networks (2011)

72. Gal, N., Stoicu-Tivadar, V.: Simulation of Medical Image Interpretation. INES 2011 15th International Conference on Intelligent Engineering Systems, pp. 33-37 (2011)

73. Balasooriya, U., Perera, M. U. S.: Intelligent Brain Hemorrhage Diagnosis System. IEEE (2011) 
74. Adam, S.Y., Yousif, A., Bakri, M.: Classification of Ischemic Stroke using Machine Learning Algorithms (2016)

75. Dimililer, K., Ïlhan, A.: Effect of image enhancement on MRI brain images with neural networks. Vienna, Austria, 12th International Conference on Application of Fuzzy Systems and Soft Computing (ICAFS 2016), pp. 39-44 (2016)

76. Burcu-Toprak, I., Fatih-Caglar, M., Merdan, M.: Automatic Recognition of Epilepsy from EEG using Artificial Neural Network and Discrete Wavelet Transform (2007)

77. RadiologyInfo.org para pacientes. Disponible en: http://www.radiologyinfo.org/sp/ info.cfm?pg=ultrasound-cranial

78. Zhanyu, M., Zheng-Hua, T., Jun, G.: Feature selection for neutral vector in EEG signal classification. Neurocomputing, pp. 937-945 (2016)

79. Dridi, M., Bouallegue, B., Ali-Hajjaji, M., Mtibaa, A.: An Enhencment Medical Image Compression Algorithm Based on Neural Network. International Journal of Advanced Computer Science and Applications, Vol. 7, No. 5 (2016)

80. García, C.: Análisis de la deformación craneal por aplicación de esfuerzos. Instituto Politécnico Nacional, Centro de Investigación en Computación (2013)

81. Sharma, N., Lalit, M.: Automated medical image segmentation techniques. India, Journal of Medical Physic, Vol. 35, No. 1, pp. 3-14 (2010)

82. Cárdenas, S. Y.: Implementación y Evaluación de Redes Neuronales Artificiales tipo "Pulse-Coupled Neural Networks" (PCNN) aplicadas a Visión Artificial. CENIDET, Cuernavaca, Morelos, México (2015)

83. Rastgarpour, M., Shanbehzadeh, J.: Application of AI Techniques in Medical Image Segmentation and Novel Categorization of Available Methods and Tools. In: Proceedings of the International MultiConference of Engineers and Computer Scientists, Vol. I, Iran, (2011)

84. Gordillo, N., Montseny, E., Sobrevilla, P.: State of the art survey on MRI brain tumor segmentation. México, Magnetic Resonance Imaging, pp. 1426-1438 (2013)

85. Lay-Khoo, L., Siau-Chuin, L.: A Survey of Medical Image Processing Tools. In: 2015 4th International Conference on Software Engineering and Computer Systems (ICSECS), pp. $171-176(2015)$

86. Rodrigues, P. M., Teixeira, J. P.: Classification of Electroencephalogram Signals Using Artificial Neural Networks. In: 2010 3rd International Conference on Biomedical Engineering and Informatics (2010) 